Pacific Journal of Mathematics

EXTENSION PROPERTIES INDUCED BY COMPLETE 


\title{
EXTENSION PROPERTIES INDUCED BY COMPLETE QUASI-UNIFORMITIES
}

\author{
Hans-Peter Kunzi and Peter Fletcher
}

\begin{abstract}
We consider the completeness of the following members of the Pervin quasi-proximity class of a completely regular Hausdorff space: $\mathscr{P} \mathscr{F}, \mathscr{L} \mathscr{F}, \mathscr{S} \mathscr{C}, \mathscr{F} \mathscr{T}$ and $\mathscr{F} \mathscr{G} \mathscr{N} \mathscr{E}$. We show that these completeness properties are extension properties, as defined by R. G. Woods, which for $\mathscr{P} \mathscr{F}, \mathscr{L} \mathscr{F}$ and $\mathscr{S} \mathscr{C}$ are closely related to almost realcompactness. Indeed, in a countably paracompact space of non-measurable cardinality, PF-completeness, LF-completeness, SC-completeness and almost realcompactness coincide. We show that the fine quasi-uniformity of any $\Sigma$-product with compact factors is almost precompact, and it follows that no $\Sigma$-product is FINE-complete. If a $\Sigma$-product is $C^{*}$-embedded in its Tychonoff product $\pi$, and if $\pi$ is $P$-complete for any of the completeness properties under consideration, then $\pi$ is the maximal $P$-extension of $\Sigma$.
\end{abstract}

1. Introduction. Although every topological space admits a quasiuniformity, in this paper all spaces are presumed to be completely regular Hausdorff spaces. A major portion of Chapter 3 of [13] is devoted to the study of the point-finite, locally finite, semi-continuous, fine-transitive and fine quasi-uniformities, which we denote, as in [13], by $\mathscr{P} \mathscr{F}, \mathscr{L} \mathscr{F}$, $\mathscr{S} \mathscr{C}, \mathscr{F} \mathscr{T}$ and $\mathscr{F} \mathscr{I} \mathscr{N} \mathscr{E}$. Necessary and sufficient conditions for these quasi-uniformities to be precompact are given in [13, page 58]. The companion problem of determining necessary and sufficient conditions for these quasi-uniformities to be complete is indicated in [13, page 59] but left unresolved; and it is clear that topological characterizations of the completeness of these quasi-uniformities are in general hard to formulate. Indeed, although the authors have recently shown that a separable locally compact normal space due to S. P. Franklin and M. Rajagopalan admits no complete quasi-uniformity, it is still unanswered whether $\mathscr{F} \mathscr{T}$ is complete whenever $\mathscr{F} \mathscr{I} \mathscr{N} \mathscr{E}$ is complete.

Our first proposition establishes that each of the completeness properties discussed above is an extension property as defined by R. G. Woods [53]. Accordingly, by results of H. Herrlich and J. van der Slot [24], if $\mathscr{P}$ is any of the completeness properties under consideration, each space $X$ has a unique maximal $P$-extension $\mathscr{P}(X)$ with the following properties:

(1) $\mathscr{P}(X)$ has property $\mathscr{P}$.

(2) $X$ is a dense subspace of $\mathscr{P}(X)$. 
(3) If $f: X \rightarrow Y$ is a continuous map from $X$ into a space $Y$ satisfying property $\mathscr{P}$, then $f$ admits a continuous extension $g: \mathscr{P}(X) \rightarrow Y$.

The space $\mathscr{P}(X)$ is characterized as the intersection of all subspaces of $\beta X$ that have property $\mathscr{P}$ and contain $X$.

In a countably paracompact space of non-measurable cardinality, PF-completeness, LF-completeness, SC-completeness, and almost realcompactness coincide. On the other hand, any weakly orthocompact space (e.g. $\left.\omega_{1}\right)$ is FT-complete. We show that no $\Sigma$-product is FINE-complete. Moreover, for any completeness property $\mathscr{P}$ under consideration, if a $\Sigma$-product $X$ is $C^{*}$-embedded in the Tychonoff product and the Tychonoff product is $P$-complete, then the ordinary product is $\mathscr{P}(X)$. For example, by $\mathrm{H}$. Corson's well-known result [5, Theorem 2], if $\Sigma$ is a $\Sigma$-product of separable metrizable spaces and $\pi$ is the corresponding product of these spaces, then $\pi$ is the maximal FINE-extension of $\Sigma$.

A space $X$ is said to be $P$-pseudocompact provided that $\mathscr{P}(X)=\beta X$ [53]. The problem of characterizing $P$-pseudocompactness, which is related to the problem of characterizing the extension property $\mathscr{P}$, usually proves to be a somewhat more tractable problem. We show that if $\mathscr{P}$ is PF-completeness, LF-completeness, or SC-completeness, then $P$-pseudocompactness is the property that the corresponding quasi-uniformity is almost precompact. (A quasi-uniformity $\mathscr{U}$ on a topological space $X$ is almost precompact provided that for each $U \in \mathscr{U}$ there is a finite set $F$ so that $\overline{U(F)}=X$.) Although we have no counterexample, it seems unlikely that the fine quasi-uniformity is almost precompact for every space that is $P$-pseudocompact for $\mathscr{P}=$ FINE-completeness. We show that for $\mathscr{P}=$ FINE-completeness (or FT-completeness), FINE (FT)-almost precompactness implies $P$-pseudocompactness and satisfies the requirements indicated by Woods in [53, Proposition 2.2] and by S. Broverman in [3, Theorem 2.3], which are necessary in order that almost precompactness be $P$-pseudocompactness. Finally, we show that if $p$ is a point in the remainder of $\beta \omega$, then $\beta \omega-\{p\}$ is almost precompact with respect to the fine quasi-uniformity.

Throughout, we adopt the notation of [53] to describe extension properties and of [13] to describe quasi-uniform space properties. Whenever possible we cite from these sources, rather than from the original source; the reader may consult these sources in order to determine the origin of a result. Moreover, the reader will find that familiarity with those two sources is essential to his full understanding of the present paper. In particular, we have need for the following basic concepts, which are discussed in more detail in [13]. A relation $V$ on a space $X$ is an (open) 
neighbornet provided that, for each $x \in X, V(x)$ is an (open) neighborhood of $x$. A neighbornet $V$ is normal provided that there is a sequence $\left\langle V_{n}\right\rangle$ of neighbornets so that, for each $n \in N, V_{n+1}^{2} \subset V_{n}$ and $V_{1} \subset V$. Note that every transitive neighbornet is an open normal neighbornet and that a neighbornet belongs to $\mathscr{F} \mathscr{J} \mathscr{N} \mathscr{E}$ if and only if it is a normal neighbornet. A space $X$ is orthocompact (quasi-normal) provided that for each open cover $\mathscr{C}$ of $X$ there is a transitive (normal) neighbornet $V$ so that $\{V(x): x \in X\}$ refines $\mathscr{C}$. A space $X$ is weakly orthocompact (weakly quasi-normal) provided that neighbornets, as above, exist for each directed open cover of $X$.

2. Completeness. In this section we show that the completeness properties discussed in this paper are extension properties, and we relate these properties to the well-known extension properties, (almost) realcompactness and Dieudonné completeness. We show that FINE-completeness and FT-completeness are preserved by continuous perfect maps; the corresponding result for PF-completeness is an open question.

A filter $\mathscr{F}$ on a quasi-uniform space $(X, \mathscr{U})$ is a $\mathscr{U}$-Cauchy filter provided that for each $U \in \mathscr{U}$ there exists a $p \in X$ so that $U(p) \in \mathscr{F}$, and $(X, \mathscr{U})($ or $\mathscr{U})$ is said to be complete provided that every $\mathscr{U}$-Cauchy filter has a cluster point. Since all the quasi-uniformities discussed herein belong to the Pervin quasi-proximity class, which is locally symmetric, every Cauchy filter under consideration that has a cluster point has a unique cluster point, to which it converges [13, Proposition 3.9]. We do not consider the Pervin quasi-uniformity, itself, since the Pervin quasi-uniformity of a space $X$ is complete if and only if $X$ is compact.

Proposition 2.1. Let $\mathscr{P}$ be the property that a topological space $X$ satisfies provided that $X$ is complete with respect to the point-finite, locally finite, semi-continuous, FINE-transitive, or fine quasi-uniformity. Then $\mathscr{P}$ is an extension property.

Proof. By a result of Herrlich and van der Slot, it suffices to show that $\mathscr{P}$ is a closed-hereditary productive property that is satisfied by all compact spaces [24, Theorem 1]. Since every quasi-uniformity of a compact space is complete, $\mathscr{P}$ is satisfied by all compact spaces. Moreover, by [13, Proposition 3.10 and remarks in $\S 2.18], \mathscr{P}$ is closed hereditary. To show that $\mathscr{P}$ is productive we consider as typical the case that $\mathscr{P}$ is PF-completeness. Let $X=\Pi\left\{X_{\alpha}: \alpha \in A\right\}$ be a product of PF-complete spaces, for each $\alpha \in A$ let $\mathscr{U}_{\alpha}$ be the point-finite covering quasi-uniformity of $X_{\alpha}$, and let $\mathscr{U}$ be the point-finite covering quasi-uniformity of $X$. Then 
$\Pi\left\{\mathscr{U}_{\alpha}: \alpha \in A\right\}$ is a complete quasi-uniformity; and, since $\mathscr{U}$ contains $\Pi\left\{\mathscr{U}_{\alpha}: \alpha \in A\right\}, \mathscr{U}$ is complete as well.

Let us recall that a topological space $X$ is almost realcompact [17], (closed complete [9]) provided that if $\mathscr{F}$ is an open (closed) ultrafilter that has no cluster point, there is a countable subcollection $\mathscr{F}^{\prime}$ of $\mathscr{F}$ so that $\bigcap\left\{\bar{F}: F \in \mathscr{F}^{\prime}\right\}=\varnothing$.

Proposition 2.2. [8, Theorem 1.7] and [14, Corollary to Theorem 5.1]. Let $X$ be a topological space. Then the following statements are equivalent.

(a) $X$ is almost realcompact.

(b) $X$ is the perfect image of a realcompact space.

(c) $(X, \mathscr{S} \mathscr{C})$ is a complete quasi-uniform space.

We say that a space $X$ is Dieudonne complete provided that the fine uniformity of $X$ is complete. The terms PF-complete, LF-complete, SCcomplete, and so on are similar abbreviations; e.g., a space $X$ is PF-complete provided that $\mathscr{P} \mathscr{F}$ is a complete quasi-uniformity for $X$. In the diagrams to follow, which indicate the relationships that hold among the completeness properties we are to study, the symbol $\stackrel{*}{\rightarrow}$ indicates an implication that holds for spaces that have no closed discrete subspaces of measurable cardinality. The non-trivial implications given in the first diagram are either well known or are consequences of Propositions $2.2-2.4$.

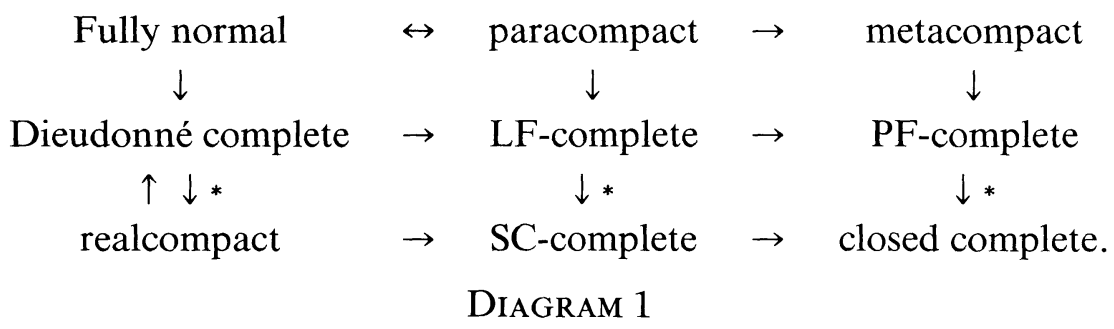

Proposition 2.3. Let $X$ be a topological space.

(a) If $X$ is Dieudonné complete, $X$ is LF-complete.

(b) If $X$ is metacompact or LF-complete, $X$ is PF-complete.

(c) If $X$ is SC-complete, $X$ is closed complete.

Proof. (a) Assume that $X$ is Dieudonné complete and let $\mathscr{U}$ denote the fine uniformity of $X$. Then $\mathscr{U} \subset \mathscr{L} \mathscr{F}[13$, Proposition 5.3] and so if $X$ is Dieudonné complete, $X$ is obviously LF-complete. (b) Since a $\mathscr{P} \mathscr{F}$-Cauchy filter meets every point-finite open cover, it is obvious that every metacompact space is PF-complete. The proof that every LF-complete space is 
PF-complete follows as in the proof of statement (a). (c) This implication is the well-known result $[9$, Theorem 1.6].

The proof of the following proposition uses ideas of $P$. Zenor [56].

Proposition 2.4. Let $X$ be a topological space that has no closed discrete subspace of measurable cardinality.

(a) If $X$ is LF-complete, $X$ is SC-complete.

(b) If $X$ is $\mathrm{PF}$-complete, $X$ is closed complete.

(c) If $X$ is normal and $\mathrm{PF}$-complete, $X$ is realcompact.

Proof. (a) Assume that $X$ is LF-complete and let $\mathscr{U}$ be an ultrafilter on $X$ that has no cluster point. We show that $\mathscr{U}$ is not an $\mathscr{S} \mathscr{C}$-Cauchy filter. Since $\mathscr{U}$ is not an $\mathscr{L} \mathscr{F}$-Cauchy filter, there is a locally finite open cover $\mathscr{G}$ of $X$ so that $\mathscr{G} \cap \mathscr{U}=\varnothing$. There is a set $D$, necessarily infinite, so that $\{\operatorname{st}(x, \mathscr{G}): x \in D\}$ covers $X$ and no member of $\mathscr{G}$ contains two points of $D$, [37, Theorem 18]. Set $Y=\{G \in \mathscr{G}: G \cap D \neq \varnothing\}$. For each $U \in \mathscr{U}$, set $M(U)=\{G \in Y: U \cap G \neq \varnothing\}$. Then $\mathscr{M}=\{M(U): U \in \mathscr{U}\}$ is a filter base on $Y$ and there is an ultrafilter $\mathscr{V}$ on $Y$ containing $\mathscr{M}$. Since $\operatorname{card}(Y)=\operatorname{card}(D)$, which is a non-measurable cardinal, $Y$ with the discrete topology is realcompact. Since $\mathscr{G} \cap \mathscr{U}=\varnothing, \cap \mathscr{V}=\varnothing$ and so there is a countable subcollection $\left\{K_{n}: n \in \omega\right\}$ of $\mathscr{V}$ so that $\bigcap\left\{K_{n}: n \in \omega\right\}=\varnothing$. We assume, without loss of generality, that $K_{n+1} \subset K_{n}$ for each $n \in \omega$. For each $n \in \omega$, set $F_{n}=X-\bigcup K_{n}$. Let $n \in \omega$ and suppose that $F_{n} \in \mathscr{U}$. Then $\varnothing=M\left(F_{n}\right) \cap K_{n} \in \mathscr{V}-$ a contradiction. Therefore, for each $n \in \omega$, $X-\overline{\cup K_{n}} \notin \mathscr{U}$. Suppose that $\mathscr{C}=\left\{X-\overline{\cup K_{n}}: n \in \omega\right\}$ covers $X$. For each positive integer $n$ set $G_{n}=X-\overline{\cup K_{n}}$ and for each non-positive integer $n$ set $G_{n}=\varnothing$. Then $\left\{G_{n}: n\right.$ is an integer $\}$ is an open spectrum and so if $\mathscr{U}$ is an $\mathscr{S} \mathscr{C}$-Cauchy filter there is a positive integer $n$ so that $G_{n} \in \mathscr{U}$ [13, Theorem 2.12]. Thus, in order to show that $\mathscr{U}$ is not an $\mathscr{S} \mathscr{C}$-Cauchy filter, it suffices to show that $\left\{X-\overline{\cup K_{n}}: n \in \omega\right\}$ covers $X$. Suppose that $y \notin \bigcup\left\{X-\overline{\cup K_{n}}: n \in \omega\right\}$. For each $n \in \omega, K_{n}$ is locally finite and hence closure preserving, and so there is a $V_{n} \in K_{n}$ so that $y \in \bar{V}_{n}$. But as $\mathscr{G}$ is locally finite, $y$ belongs to the closures of only finitely many members of $\mathscr{G}$. Thus, there exists $m \in \omega$ so that $V_{n}=V_{m}$ for infinitely many $n$. It follows that $V_{m} \in \bigcap\left\{K_{n}: n \in \omega\right\}-$ a contradiction.

(b) The proof of this assertion requires only minor modifications of the proof above. Assume that $X$ is PF-complete and let $\mathscr{F}$ be a free closed ultrafilter on $X$. Then $\mathscr{F}$ is contained in an ultrafilter $\mathscr{U}$ and $\mathscr{F}=\{\bar{U}$ : $U \in \mathscr{U}\}$. As above, there are subcollections $\left\{K_{n}: n \in \omega\right\}$ of a point-finite open cover $\mathscr{G}$ so that $\bigcap\left\{K_{n}: n \in \omega\right\}=\varnothing$ and $K_{n+1} \subset K_{n}$ for each $n \in \omega$. 
For each $n \in \omega, X-\cup K_{n} \notin \mathscr{F}$ and so there exists $A_{n} \in \mathscr{F}$ so that $A_{n} \subset \cup K_{n}$. Thus, we have that $\bigcap\left\{A_{n}: n \in \omega\right\} \subset \bigcap\left\{\cup K_{n}: n \in \omega\right\}=\varnothing$.

(c) The proof of this assertion requires only minor modifications of the proof of assertion (b). Assume that $X$ is normal and PF-complete and let $\mathscr{Z}$ be a free $\mathscr{Z}$-ultrafilter. Then $\mathscr{Z}$ is contained in a closed ultrafilter $\mathscr{F}$, which in turn is contained in an ultrafilter $\mathscr{U}$. We note that $\mathscr{F}=\{\bar{U}$ : $U \in \mathscr{U}\}$. Since $\mathscr{U}$ is not a $\mathscr{P} \mathscr{F}$ Cauchy filter, $\{X-F: F \in \mathscr{F}\}$ has a point-finite open refinement $\mathscr{G}$. As above, there exist open sets $G_{n}$ so that $\bigcap\left\{G_{n}: n \in \omega\right\}=\varnothing$ and, for each $n \in \omega, X-G_{n} \notin \mathscr{F}$. Thus, there exists $\left\{A_{n}: n \in \omega\right\} \subset \mathscr{F}$ so that $A_{n} \subset G_{n}$ for each $n \in \omega$. Since $X$ is normal, there are zero-sets $Z_{n}$ so that $A_{n} \subset Z_{n} \subset G_{n}$ for each $n \in \omega$. Then $\left\{Z_{n}\right.$ : $n \in \omega\}$ is a countable free subcollection of $\mathscr{Z}$.

It is evident that a discrete space of measurable cardinality satisfies all the conditions of the top line of Diagram 1 and none of the conditions of the bottom line. Thus the hypothesis of Proposition 2.4 that no closed discrete subspace of $X$ be of measurable cardinality cannot be omitted. In the presence of further restrictions upon the space $X$, several other implications among the completeness properties of Diagram 1 may be obtained. We begin by considering properties related to normality. A space $X$ is almost 2-fully normal provided that the collection of all neighborhoods of the diagonal of $X$ is the fine uniformity of $X$, and $X$ is somewhat normal provided that, for each open cover $\mathscr{C}$ of $X,\{\operatorname{st}(x, \mathscr{C}): x \in X\}$ is a normal cover of $X$. It is known that an almost 2-fully normal space is both collectionwise normal and somewhat normal [36, Theorem 2.9] and [11, Page 4]. A somewhat normal space need not be normal [31, Example 2.6].

Proposition 2.5. Every PF-complete space that is somewhat normal or collectionwise normal is Dieudonné complete.

Proof. Let $X$ be a PF-complete somewhat normal space, let $\mathscr{F}$ be an ultrafilter that is Cauchy with respect to the fine uniformity, and let $\mathscr{G}$ be a point-finite open cover of $X$. Since $\mathscr{G}^{*}=\{\operatorname{st}(x, \mathscr{G}): x \in X\}$ is a normal cover, $\mathscr{G}^{*}$ and $\mathscr{G}$ meet $\mathscr{F}$. Hence $\mathscr{F}$ is a $\mathscr{P} \mathscr{F}$ Cauchy filter and converges. The remaining implication of the proposition is an immediate consequence of the well-known result of E. Michael that every point-finite open cover of a collectionwise normal space is normal.

Proposition 2.6. Every normal LF-complete space is Dieudonné complete.

Proof. In a normal space, every locally finite open cover is normal. 
Let $X$ denote Mary Ellen Rudin's Dowker space. By results of K. P. Hart [21] and P. Simon [44], $X$ is an almost 2-fully normal closed-complete space that is not SC-complete. Consequently, $X$ is not PF-complete. In [17, Theorem 11], Z. Frolik asserts that every almost realcompact normal space is realcompact, but, as P. Kenderov points out [29], Frolik's proof has a gap. Frolik proves only that every countably paracompact almost realcompact normal space is realcompact. The truth of Frolik's original assertion remains an open question, to which the following questions are closely related.

Question 2.7. Is every SC-complete space PF-complete?

Question 2.8. Is every PF-complete space of non-measurable cardinality SC-complete?

Proposition 2.9. A countably orthocompact separable space is PF-complete if and only if it is SC-complete.

Proof. Let $X$ be a countably orthocompact separable SC-complete space and let $\mathscr{F}$ be a $\mathscr{P} \mathscr{F}$ Cauchy ultrafilter over $X$. By [13, Proposition 5.13], $X$ is countably metacompact. An argument similar to that given in the proof of Proposition 2.4 (a) establishes that an ultrafilter $\mathscr{F}$ is an $\mathscr{S} \mathscr{C}$-Cauchy filter if and only if it meets each countable open cover. Since every countable open cover of $X$ has a point-finite open refinement, $\mathscr{F}$ is an $\mathscr{S} \mathscr{C}$-Cauchy filter and so converges. The other direction is immediate, since in a separable space every point-finite open cover is countable.

The method of proof of Proposition 2.9 yields the following corollary.

COROLlARY. Every countably paracompact SC-complete space is LFcomplete.

EXAMPLE 2.10. The Dieudonné Plank is an almost realcompact space that is not LF-complete. Let $X=\left(\omega_{1}+1\right) \times(\omega+1)-\left\{\left(\omega_{1}, \omega\right)\right\}$. For each $(p, q) \in \omega_{1} \times \omega$ define $H(p, q)=\left\{(x, q): p \leq x \leq \omega_{1}\right\}$ and define $V(p, q)=\{(p, y): q \leq y \leq \omega\}$. The topology under consideration is the one for which $\left\{H(p, q), V(p, q):(p, q) \in \omega_{1} \times \omega\right\}$ is a subbase. It is well known that $X$ is almost realcompact [27]. To see that $X$ is not LF-complete, let $\mathscr{U}$ be an ultrafilter on $X$ containing $\left\{\left(\alpha, \omega_{1}\right) \times[n, \omega)\right.$ : $\left.\alpha \in \omega_{1}, n \in \omega\right\}$. Let $\mathscr{C}$ be a locally finite open cover of $X$ and for each $n \in \omega$ let $\beta_{n} \in \omega$, so that $H\left(\beta_{n}, n\right)$ is a subset of some member of $\mathscr{C}$. Let $\beta=\sup \left\{\beta_{n}: n \in \omega\right\}$. Suppose that there is no finite subcollection of $\mathscr{C}$ 
covering $\left(\beta, \omega_{1}\right) \times[0, \omega)$. Then $\mathscr{C}$ is not locally finite at $\left(\beta+1, \omega_{1}\right)$. Therefore $\mathscr{C} \cap \mathscr{U} \neq \varnothing$ and so $\mathscr{U}$ is an $\mathscr{L} \mathscr{F}$-Cauchy filter without a cluster point.

In the preceding corollary, countable paracompactness cannot be replaced by countable metacompactness, because the Dieudonné Plank is a metacompact (and hence PF-complete) SC-complete space that we have just seen is not LF-complete. In light of $K$. Hardy's result that a countably paracompact closed-complete space is almost realcompact [20], the following diagram indicates the relationships among the completeness properties under consideration that obtain in countably paracompact spaces.

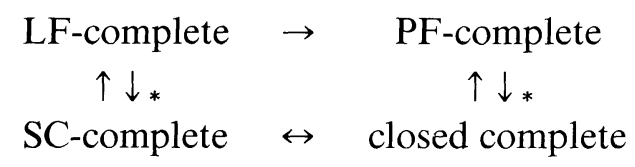

\section{Diagram 2}

Diagram 2 suggests the conjecture that every countably paracompact PF-complete space is LF-complete. Somewhat surprizingly, this conjecture is known to be false, for $R$. Haydon has given an example of a normal metacompact space (of measurable cardinality) that is not Dieudonné complete [22, Remark following Example 3.1]. It follows from Proposition 2.6 that Haydon's space is not LF-complete, and since the space is normal and metacompact it is obviously both countably paracompact and PFcomplete. It is also noteworthy that the only property of Diagram 2 satisfied by Mary Ellen Rudin's Dowker space is closed completeness.

We consider briefly the behavior of our completeness properties in the class of (weak) cb-spaces. (Weak) cb-spaces were first defined and studied by J. G. Horne [25], J. Mack [33] and J. Mack and D. Johnson [34], but we refer the reader to [49] for definitions and results. For our purposes it is enough to note that an extremally disconnected space is a weak cb-space, that a normal or weak cb-space is a cb-space if it is countably paracompact, and that a space is realcompact if it is an almost realcompact weak cb-space or a closed complete cb-space [9, Corollary 1.10]. By virtue of our previous propositions, these results justify the following diagram.

weak cb-space

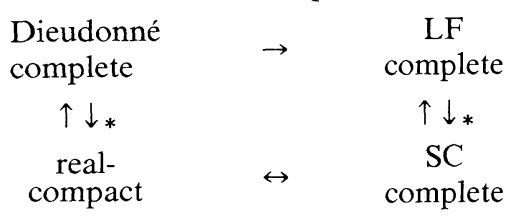

cb-space

\begin{tabular}{|c|c|c|c|c|}
\hline $\begin{array}{l}\text { ieudonné } \\
\text { mplete }\end{array}$ & $\rightarrow$ & $\begin{array}{c}\mathrm{LF} \\
\text { complete }\end{array}$ & $\rightarrow$ & $\begin{array}{c}\text { PF } \\
\text { complete }\end{array}$ \\
\hline$\uparrow \downarrow_{*}$ & & $\uparrow \downarrow *$ & & \\
\hline ompact & & $\begin{array}{c}\mathrm{SC} \\
\text { complete }\end{array}$ & $\leftrightarrow$ & \\
\hline
\end{tabular}


Evidently both parts of Diagram 3 collapse when we restrict our attention to spaces in which each closed discrete subspace is of non-measurable cardinality. Haydon's example, which is normal and countably paracompact, is a cb-space. Hence, there is a measurable cardinal if and only if there is a PF-complete cb-space that is not LF-complete. As the following question indicates, the relationship between LF-completeness and Dieudonné completeness has not yet been determined.

Question 2.11. Is every LF-complete (cb-) space Dieudonné complete?

It follows from Corollary 2.9 that Question 2.11 would be answered negatively if there exists a countably paracompact almost realcompact space of non-measurable cardinality that is not realcompact. Unfortunately, the existence of such a space is itself an unsolved problem. The next proposition provides a class of weak cb-spaces in which LF-completeness and Dieudonné completeness coincide. The proof of this proposition, and of several propositions to follow, is facilitated by the simple observation that a regular quasi-uniform space is complete provided that every open ultrafilter that is a Cauchy filter has a cluster point [15, Theorem 3.6].

PROPOSITION 2.12. Every extremally disconnected LF-complete space is Dieudonné complete.

Proof. Let $X$ be an extremally disconnected LF-complete space and let $\mathscr{U}$ be an open ultrafilter over $X$ that has no cluster point. There is a locally finite open cover $\mathscr{L}$ so that $\mathscr{L} \cap \mathscr{U}=\varnothing$. For each $L \in \mathscr{L}$, there is $U(L) \in \mathscr{U}$ so that $\bar{L} \cap \overline{U(L)}=\varnothing$. Since $\mathscr{G}=\{\bar{L}: L \in \mathscr{L}\}$ is a locally finite cover of $X$ by open-and-closed sets, $\mathscr{G}$ is a normal cover of $X$ that misses $\mathscr{U}$. Thus $\mathscr{U}$ is not a Cauchy filter with respect to the fine uniformity.

We now consider the behavior of FT-completeness and FINE-completeness. Since $\mathscr{S} \mathscr{C} \subset \mathscr{F} \mathscr{T}$, it follows from Proposition 2.2 that every almost realcompact space is FT-complete and hence FINE-complete. The implications of Diagram 4, which are evident, indicate, however, that FT-completeness and FINE-completeness are far weaker properties than almost realcompactness.

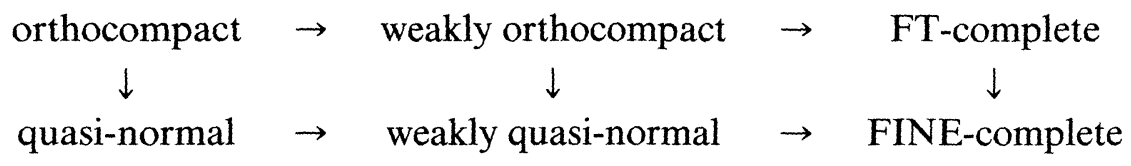


It is unknown whether every FINE-complete space is FT-complete. Indeed, if $X$ is an $F_{\sigma}$-discrete space or an orthocompact semi-stratifiable space, then $\mathscr{F} \mathscr{T}(X)=\mathscr{F} \mathscr{I} \mathscr{N} \mathscr{E}(X)$; in general it appears difficult to find spaces in which $\mathscr{F} \mathscr{T}$ and $\mathscr{F} \mathscr{I} \mathscr{N} \mathscr{E}$ differ (see [13, Chapter 6]). As noted in [30], Example 7.25 of [13] provides a space that is quasi-normal but not weakly orthocompact. Example 5.34 of [13] is FT-complete but not weakly quasi-normal and $\omega_{1} \times \beta \omega_{1}$ is a weakly orthocompact space that is not quasi-normal [13, Corollary 5.40]. Thus none of the remaining implications of Diagram 4 is reversible, (and it seems unlikely that the implication FT-complete $\rightarrow$ FINE-complete will turn out to be reversible either.)

We end this section by considering the behavior with respect to perfect continuous maps of the completeness properties we have been considering. It is shown in [13] that weak orthocompactness and weak quasi-normality are inverse invariant under perfect continuous maps (as are the extension properties FT-completeness and FINE-completeness). The proof given below that FINE-completeness is preserved by perfect continuous maps can be modified to show that FT-completeness, weak quasi-normality and weak orthocompactness [42, Lemma 2.4] are also preserved by such maps. Thus, although neither orthocompactness nor quasi-normality is preserved by perfect continuous maps [13, Example 5.24], the remaining properties of Diagram 4 are invariant under perfect continuous maps in both directions.

Proposition 2.13. FINE-completeness is preserved by perfect continuous maps.

Proof. Let $f: X \rightarrow Y$ be a perfect continuous map from a FINE-complete space $X$ onto a space $Y$ and let $\mathscr{F}$ be a $\mathscr{F} \mathscr{I} \mathscr{N} \mathscr{E}(Y)$-Cauchy filter. Then $\left\{f^{-1}(F): F \in \mathscr{F}\right\}$ is contained in an ultrafilter $\mathscr{U}$ on $X$. Let $V$ be a normal neighbornet of $X$ and let $W$ be a normal neighbornet of $X$ so that $W^{2} \subset V$. Set $W^{*}=\left\{(a, b) \in Y \times Y: f^{-1}(b) \subset W\left(f^{-1}(a)\right)\right\}$. Since $f$ is closed, $W^{*}$ is a normal neighbornet of $Y$ and there exists $y \in Y$ so that $W^{*}(y) \in \mathscr{F}$. There is a finite subset $A$ of $X$ so that $f^{-1}(y) \subset W(A)$. Since $f^{-1}\left(W^{*}(y)\right) \subset W\left(f^{-1}(y)\right) \subset W^{2}(A) \subset V(A)$ and $f^{-1}\left(W^{*}(y)\right) \in \mathscr{U}$, there exists $a \in A$ such that $V(a) \in \mathscr{U}$. Thus $\mathscr{U}$ is a $\mathscr{F} \mathscr{I} \mathscr{N} \mathscr{E}(X)$-Cauchy filter and converges, say to $x$. It is easily seen that $f(x)$ is a cluster point of $\mathscr{F}$.

It is interesting to note that the absolute of the Dieudonné Plank is realcompact (and hence LF-complete) [50, Example 1], for it follows that 
LF-completeness is not preserved by perfect continuous maps. Because almost realcompactness is preserved by perfect continuous maps, the following question is obviously related to Questions 2.7 and 2.8.

Question 2.14. Is PF-completeness preserved by perfect continuous maps?

The results given above show that FT-completeness and FINE-completeness are extremely general extension properties. Nonetheless, in the next sections we determine some interesting topological spaces that are not FINE-complete.

3. Almost precompactness and $P$-pseudocompactness. In 1971 , the second author and S. A. Naimpally introduced almost precompactness and proved for an arbitrary Hausdorff space $X$ that $(X, \mathscr{S} \mathscr{C})$ is almost precompact if and only if $X$ is countably almost compact [15, Theorem 4.1]. J. R. Porter and R. G. Woods have pointed out that if $\mathscr{P}$ is the property, almost realcompactness, then countable almost compactness is the natural analogue in the class of Hausdorff spaces of $P$-pseudocompactness [40, Theorem 3.19]. Furthermore, a space $X$ is compact if either of the following obtains: There is an extension property $\mathscr{P}$ so that $X$ satisfies $\mathscr{P}$ and is $P$-pseudocompact, or there is a compatible complete quasi-uniformity that is almost precompact. Thus it is reasonable to suppose that for some completeness properties $\mathscr{P}$ a space is $P$-pseudocompact only if the corresponding quasi-uniformity is almost precompact. In this section, we pursue the connection between $P$-pseudocompactness and almost precompactness that Porter and Woods's theorem suggests. We adopt the self-explanatory terminology PF-pseudocompactness, LF-pseudocompactness and so forth, and we recall the easy but useful observation that a quasi-uniform space $(X, \mathscr{U})$ is almost precompact if and only if every open ultrafilter over $X$ is a Cauchy filter [15].

LeMMA 3.1. Let $X$ be a space and let $\mathscr{P}=\mathscr{P} \mathscr{F}, \mathscr{L} \mathscr{F}, \mathscr{S} \mathscr{C}, \mathscr{F} \mathscr{T}$, or $\mathscr{F} \mathscr{I} \mathscr{N} \mathscr{E}$. If $(X, \mathscr{P})$ is almost precompact, $X$ is P-pseudocompact.

Proof. By definition, $X \subset \mathscr{P}(X) \subset \beta X$. Let $\mathscr{G}$ be an open ultrafilter over $X$. Since $(X, \mathscr{P})$ is almost precompact, $\mathscr{G}$ is a $\mathscr{P}$-Cauchy filter and the extension of $\mathscr{G}$ to $\mathscr{P}(X)$ is an open $\mathscr{P}$-Cauchy filter on $\mathscr{P}(X)$. It follows that every open filter on $X$ has a cluster point in $\mathscr{P}(X)$, and so $\mathscr{P}(X)$ is compact [15, Theorem 3.5]. 
Proposition 3.2. Let $X$ be a space and let $\mathscr{U}=\mathscr{P} \mathscr{F}, \mathscr{L} \mathscr{F}$, or $\mathscr{S} \mathscr{C}$.

Then the following statements are equivalent.

(a) $X$ is $U$-pseudocompact.

(b) $(X, \mathscr{U})$ is almost precompact.

(c) $X$ is pseudocompact.

Proof. By [15, Theorem 4.1], $(X, \mathscr{S} \mathscr{C})$ is almost precompact if and only if $X$ is pseudocompact. Moreover, as the maximal SC-extension of $X$ is the almost realcompactification $a X$ of $X$ [55], if $X$ is SC-pseudocompact, then $a X=\beta X$ and so $X$ is pseudocompact. In light of the previous lemma, it suffices to establish the following implications: (i) if $X$ is PF-pseudocompact, or LF-pseudocompact, $X$ is pseudocompact, (ii) if $X$ is pseudocompact, $(X, \mathscr{L} \mathscr{F})$ is almost precompact, and (iii) if $(X, \mathscr{L} \mathscr{F})$ is almost precompact, $(X, \mathscr{P} \mathscr{F})$ is almost precompact. Since $\mathscr{P} \mathscr{F}$ contains $\mathscr{L} \mathscr{F}$, which contains the fine uniformity, condition (i) obtains. Implication (ii) follows from [13, Proposition 3.19].

The proof of the remaining implication is based upon an argument of B. M. Scott [43, Theorem 1]. Let $V \in \mathscr{P} \mathscr{F}$. Without loss of generality we assume that there is a point-finite open cover $\mathscr{C}$ of $X$ so that, for each $x \in X, V(x)=\bigcap\{C \in \mathscr{C}: x \in C\}$. Let $\mathscr{V}=\{V(x): x \in X\}$. Let $\mathscr{H}$ be a family of nonempty open sets maximal with respect to the following property: Each member of $\mathscr{H}$ meets only finitely many members of $\mathscr{V}$ and no member of $\mathscr{V}$ meets two members of $\mathscr{H}$. By (i), $X$ is pseudocompact and so the point-finite open cover $\mathscr{V}$ is locally finite on a dense set. It follows that $\mathscr{H}$ is a nonempty disjoint finite family of open sets. Let $\mathscr{V}^{\prime}=\{V \in \mathscr{V}:$ for some $H \in \mathscr{H}, V \cap H \neq \varnothing\}$. For each $V \in \mathscr{V}^{\prime}$, choose one $x \in X$ so that $V(x)=V$ and let $A$ denote the finite set of points so chosen. Since $\cup \mathscr{V}^{\prime}$ is dense, $X=\overline{V(A)}$.

Corollary. A space $X$ is compact if and only if it is pseudocompact and PF-complete.

We note that the previous corollary includes both the result of Scott that every pseudocompact metacompact space is compact and the classical result that every pseudocompact Dieudonné complete space is compact.

We now consider $P$-pseudocompactness and almost precompactness for $\mathscr{F} \mathscr{I} \mathscr{N} \mathscr{E}$ and $\mathscr{F} \mathscr{T}$. As in [32], we say that a topological space $X$ is almost precompact (FT-almost precompact) provided that $\mathscr{F} \mathscr{I} \mathscr{N} \mathscr{E}(\mathscr{F} \mathscr{T})$ is an almost precompact quasi-uniformity. Although the concept of almost precompactness predates the fundamental paper of S. H. Hechler [23], it is clearly kin to the following result of Hechler, which is generalized by J. E. 
Vaughan [47, Lemma 6.4]: If $\mathscr{G}$ is an open cover of a countably compact separable space $X$ and card $(\mathscr{G})$ is smaller than the smallest cardinal, usually denoted $\mathfrak{p}$, of a maximal family of subsets of $\omega$ with the strong finite intersection property, then there is a finite subfamily $\mathscr{G}^{\prime}$ of $\mathscr{G}_{\text {so }}$ that $X=\bigcup\left\{\bar{G}: G \in \mathscr{G}^{\prime}\right\}$.

It turns out that the extension property $\omega$-boundedness, considered by Woods in [51], is useful in determining spaces that are almost precompact. A space $X$ is $\omega$-bounded provided that the closure of each countable subspace is compact [19] and [28]. Following the Russian literature, we avoid the inappropriate terminology "countable chain condition" by saying a space $X$ has the Souslin property if every collection of pairwise disjoint open subsets of $X$ is countable.

Proposition 3.3. In a FINE-complete space $X$ with the Souslin property, the closure of an $\omega$-bounded subspace is compact.

Proof. The proof is by contradiction. Let $B$ be an $\omega$-bounded subspace of $X$ such that $\bar{B}$ is not compact and let $\mathscr{C}$ be an open cover of $\bar{B}$ that has no finite subfamily covering $B$. Let $\mathscr{F}$ be the open filter generated by $\{G: G$ is an open set and there exists $C \in \mathscr{C}$ so that $B-C \subset G\}$ and let $\mathscr{G}$ be an open ultrafilter over $X$ containing $\mathscr{F}$. Since $X$ is regular, $\mathscr{G}$ has no cluster point.

The contradiction is established by showing that $\mathscr{G}$ is a $\mathscr{F} \mathscr{I} \mathscr{N} \mathscr{E}$-Cauchy filter. Let $U \in \mathscr{F} \mathscr{I} \mathscr{N} \mathscr{E}$ and let $V$ be an open neighbornet such that $V^{2} \subset U$. Since $V(B)$ is an open subspace of a space with the Souslin property, there is a countable subset $D$ of $B$ so that $V(B) \subset \overline{V(D)}$. Since $\bar{D}$ is compact, there is a finite subset $E$ of $X$ so that $D \subset V(E)$. Thus $V(B) \subset \overline{V(D)} \subset \overline{V^{2}(E)} \subset \overline{U(E)}$ and $U(E) \in \mathscr{G}$. Since $E$ is finite, it follows that $\mathscr{G}$ is a $\mathscr{F} \mathscr{A} \mathscr{E}$-Cauchy filter.

The method of proof of Proposition 3.3 establishes the following corollary.

COROLlary. Let $B$ be an $\omega$-bounded subspace of a space $X$ with the Souslin property and let $V$ be an open neighbornet of $X$. Then there is a finite subset $E$ of $X$ so that $V(B) \subset \overline{V^{2}(E)}$.

A space $X$ is a weakly Lindelö space provided that every open cover of $X$ has a countable subcollection whose union is dense. A point $p$ of $X$ has countable tightness provided that if $C \subset X$ and $p \in \bar{C}$ then $p$ belongs to the closure of some countable subset of $C$. A space in which each point has countable tightness is said to be of countable tightness. 
Although the following variant of Proposition 3.3 is not used in the present paper, we believe that it may prove useful in another setting.

Proposition 3.4. Every closed $\omega$-bounded subspace of a normal weakly Lindelöf FINE-complete space is compact.

Proof. (Sketch) As in the proof of Proposition 3.3, let $B$ be a closed $\omega$-bounded subspace of a normal weakly Lindelöf FINE-complete space $X$, and define $\mathscr{C}, \mathscr{F}, \mathscr{G}, U$ and $V$ as above. To see that $\mathscr{G}$ is a $\mathscr{F} \mathscr{I} \mathscr{N} \mathscr{E}$-Cauchy filter, note that since $V(B)$ is an open set containing $B$, there is an open set $G$ so that $B \subset G \subset \bar{G} \subset V(B)$. Since $\{X-\bar{G}\} \cup\{V(b): b \in B\}$ covers $X$, there is a countable subset $D$ of $B$ so that $G \subset \overline{V(D)}$. As in the previous proof, there is a finite set $E$ so that $U(E) \in \mathscr{G}$.

COROLlaRY. Every w-bounded subspace of a normal weakly Lindelöf FINE-complete space of countable tightness is compact.

Following P. Nyikos and J. Vaughan [39] and [47], we say that a locally compact space $X$ is a Franklin-Rajagopalan space [16] provided (a) $X$ has a countable dense set of isolated points (which we identify with $\omega$ ) and (b) the remainder $X-\omega$ is homeomorphic to an ordinal with the order topology. Theorem 1.3 and Lemma 3.1 of [39] characterize a countably compact non-compact Franklin-Rajagopalan space as a space of the form $\omega \cup \mu$, where $\mu$ is an ordinal of uncountable cofinality considered to be disjoint from $\omega$, for which the topology on $\omega \cup \mu$ is defined in terms of a maximal increasing tower on $\omega$. This characterization enabled the authors to prove that every countably compact FranklinRajagopalan space of regular uncountable cardinality is almost precompact [32, Proposition 3.4]. Our corollary to Proposition 3.3 provides an alternate proof of this result, which shows that it is unnecessary to require that the Franklin-Rajagopalan space be of regular cardinality.

Proposition 3.5. Every countably compact Franklin-Rajagopalan space is almost precompact.

Proof. Let $T$ be a countably compact Franklin-Rajagopalan space. We may assume that $T$ is not compact. Thus, by the results of [39], we assume that there is an ordinal $\mu$ of uncountable cofinality so that $T=\omega \cup \mu$ (where $\omega$ and $\mu$ are considered disjoint) and an increasing maximal tower $\left\langle A_{\alpha}\right\rangle_{\alpha \in \mu}$ on $\omega$ so that the basic open neighborhoods of $T$ may be defined 
as follows. Points of $\omega$ are isolated; for $0 \leq \beta<\alpha<\mu$ and $F$ a finite subset of $\omega$ any set of the form $U(\alpha, \beta, F)=(\beta, \alpha] \cup\left[A_{\alpha}-A_{\beta}\right]-F$ is a basic open set, and any set of the form $U(0, \beta, F)=\{0\} \cup\left(A_{0}-F\right)$ is a basic open set. Let $U$ be an open normal neighbornet and let $V$ be an open neighbornet so that $V^{2} \subset U$. By the corollary to Proposition 3.3, there is a finite set $E$ so that $V(\mu) \subset \overline{V^{2}(E)}$. Since the open cover $\{V(\mu)\} \cup\{U(n): n \in \omega\}$ has a finite subcover, there is a finite subset $F$ of $\omega$ so that $T=(V(\mu) \cup U(F)) \subset \overline{U(E)} \cup U(F) \subset \overline{U(E \cup F)}$.

The proof of the following proposition makes use of the result of [7, Example 7.1] that the one-point compactification and the Stone-Čech compactification of a countably compact Franklin-Rajagopalan space coincide. In proving this proposition we also make use of a key lemma, which we will use repeatedly.

LEMMA 3.6. Let $X$ be a space with the Souslin property, let $Q$ be a FINE-complete space, and let $f: X \rightarrow Q$ be a continuous function. If $B$ is an $\omega$-bounded subset of $X$ and $x \in \mathrm{cl}_{\beta X}(B)$, then $f$ admits a continuous extension $\tilde{f}: X \cup\{x\} \rightarrow Q$.

Proof. Because FINE-completeness is a closed-hereditary property, we assume without loss of generality that $\overline{f(X)}=Q$. Let $f^{\beta}: \beta X \rightarrow \beta Q$ be the continuous extension of $f$ to $\beta X$, let $B$ be an $\omega$-bounded subset of $X$ and let $x \in \mathrm{cl}_{\beta X}(B)$. Then $f^{\beta}(x) \in \mathrm{cl}_{\beta Q} f^{\beta}(B)=\mathrm{cl}_{\beta Q} f(B)$. Note that $Q$ has the Souslin property and that $f(B)$ is an $\omega$-bounded subset of $Q$. By Proposition 3.3, $\mathrm{cl}_{Q}(f(B))$ is a compact subset of $Q$ and so this set is closed in $\beta Q$. That is, $f^{\beta}(x) \in \operatorname{cl}_{\beta Q}(f(B)) \subset \mathrm{cl}_{Q}(f(B)) \subset Q$. Hence $f^{\beta} \mid$ $X \cup\{x\}$ is the required extension of $f$.

PROPOSITION 3.7. The arbitrary product of countably compact Franklin-Rajagopalan spaces is FINE-pseudocompact.

Proof. Let $X=\Pi\left\{T_{\alpha}: \alpha \in A\right\}$ be a product of countably compact Franklin-Rajagopalan spaces and let $Y$ be the product $\Pi\left\{T_{\alpha}^{+}: \alpha \in A\right\}$ of the corresponding one-point compactifications of $T_{\alpha}$. Since each $T_{\alpha}$ is locally compact and pseudocompact, $X$ is pseudocompact and $\beta X=$ $\beta \Pi\left\{T_{\alpha}: \alpha \in A\right\}=\Pi\left\{\beta T_{\alpha}: \alpha \in A\right\}=Y[18$, Theorems 4 and 1]. It remains to show that $Y$ is the FINE-extension of $X$. Let $Q$ be a FINE-complete space and let $f: X \rightarrow Q$ be a continuous map. Let $y \in Y-X$. By 
[10, Problem 3.2A(b), p. 193], it suffices to show that $f$ admits a continuous extension $\tilde{f}: X \cup\{y\} \rightarrow Q$. Set $B=\left\{y^{\prime} \in Y: y^{\prime}(\alpha)=y(\alpha)\right.$ if $y(\alpha) \neq$ $\infty_{\alpha}$ and $0 \leq y^{\prime}(\alpha)<\mu_{\alpha}$ if $y(\alpha)=\infty_{\alpha}$, where $\mu_{\alpha}$ is the ordinal so that $\left.T_{\alpha}=\omega \cup \mu_{\alpha}\right\}$. Then $B$ is $\omega$-bounded and $y \in \operatorname{cl}_{Y}(B)$ so that by the previous lemma the required extension $\tilde{f}$ of $f$ exists.

Since every almost precompact space is pseudocompact, it is natural to ask if every almost precompact space must be countably compact. It is possible to give an easy counterexample to this conjecture, using a countably compact Franklin-Rajagopalan space of regular cardinality in place of $\omega_{1}$ in the construction of the Tychonoff Plank. The example we give here is somewhat more involved, since it makes use of remote points, but it is more interesting.

Let $X$ be a space and let $p \in \beta X$. As in [6], we say that $p$ is a remote point of $X$ and write $p \in \rho(X)$ if $p \in \beta(X)-X$, but for each nowhere dense set $A$ of $X, p \notin \operatorname{cl}_{\beta X}(A)$. We have need of the result of Woods [51, Theorem 3.5], whose proof is emended in [6, Theorem 11.1(a)], that if $X$ is a $\sigma$-compact locally compact space of countable $\pi$-weight and $X$ has no isolated points, then $\rho X$ and its complement in $\beta X-X$ are dense $\omega$-bounded subsets of $\beta X-X$.

EXAMPLE 3.8. An almost precompact space that is not countably compact. Let $X$ be the real line, or any other locally compact $\sigma$-compact non-compact space with countable $\pi$-weight and no isolated points. Let $Y=X \cup \rho(X)$. There exists an increasing sequence $\left\langle G_{n}\right\rangle$ of subsets of $X$, open in $X$, and hence in $Y$, such that each $\mathrm{cl}_{X}\left(G_{n}\right)$ is compact and such that $X=\bigcup_{n=1}^{\infty} G_{n}$. To show that $Y$ is almost precompact, let $U$ be an open normal neighbornet of $Y$ and let $V$ be an open neighbornet so that $V^{2} \subset U$. Because $X$ is separable, $Y$ has the Souslin property and so by Corollary 3.3 there is a finite set $E$ so that $V[\rho(X)] \subset \overline{V^{2}(E)} \subset \overline{U(E)}$. Set $G=Y-\overline{U(E)}$. Suppose that for each $n \in \omega, G-\bar{G}_{n} \neq \varnothing$. Then $\left\langle G-\bar{G}_{n}\right\rangle$ is a decreasing sequence of open sets and by $[6,17.1(\mathrm{~d})] Y$ is pseudocompact. Thus there is a point $p$ belonging to the closure of each $G-\bar{G}_{n}$. Since $X=\cup_{n=1}^{\infty} G_{n}, p \in \rho(X)$; but then $V(\rho(X))$ is an open set about $p$ missing $G-$ a contradiction. Therefore, there exists $n \in \omega$ such that $G \subset \bar{G}_{n}$. There is a finite set $F$ so that $\bar{G} \subset U(F)$; whence $Y$ $\subset \overline{U(E \cup F)}$.

Because $X$ is realcompact, but not compact, there is a countably infinite closed subset $A$ of $X$ that has no cluster point in $X$, and since $A$ is nowhere dense $\operatorname{cl}_{\beta X}(A) \cap \rho(X)=\varnothing$. Thus $Y$ has a countably infinite closed set without a cluster point. 
Since $\omega_{1}$ with the order topology is orthocompact [12], this space provides a simple example of a pseudocompact space that is not FT-almost precompact. Nonetheless, the following two propositions indicate that (FT-) almost precompactness mimics the behavior of pseudocompactness. In the first of these propositions conditions (a), (b), and (c) are included only for the sake of completeness. Condition (a) has been proved for the fine quasi-uniformity in [32, Proposition 2.3] (the proof given there also holds for $\mathscr{F} \mathscr{T}$ ), and we omit the proofs of (b) and (c), which are routine.

Proposition 3.9. A space $S$ is (FT-) almost precompact provided that any of the following conditions holds:

(a) $S$ is a regular-closed subspace of an (FT-) almost precompact space.

(b) $S$ is the union of finitely many (FT-) almost precompact subspaces.

(c) $S$ has an (FT-) almost precompact dense subspace.

(d) $S$ is the product of a compact space and an (FT-) almost precompact space.

Proof. Let $K$ be a compact space and let $Y$ be an (FT-) almost precompact space and suppose that $S=K \times Y$. We show that every open ultrafilter over $S$ is a Cauchy filter with respect to $\mathscr{F} \mathscr{T}(\mathscr{F} \mathscr{I} \mathscr{N} \mathscr{E})$. Let $\mathscr{U}$ be an open ultrafilter over $S$, let $\mathscr{V}=\mathscr{F} \mathscr{T}(\mathscr{F} \mathscr{I} \mathscr{N} \mathscr{E})$, and let $V \in \mathscr{V}$. Let $W$ be an open neighbornet so that $W^{2} \subset V$. For each $y \in Y$, there is a finite subset $F(y)$ of $K$ so that the compact space $K \times\{y\} \subset$ $\bigcup\{W(x, y): x \in F(y)\}$. Thus

$$
W(K \times\{y\}) \subset \bigcup\{V(x, y): x \in F(y)\} .
$$

Set

$$
\tilde{W}=\{(a, b) \in Y \times Y: K \times\{b\} \subset W(K \times\{a\})\} .
$$

Since $\pi_{Y}$ is closed, $\tilde{W}$ belongs to the fine (transitive) quasi-uniformity of $Y$, and since $Y$ is almost precompact, there exists a $p \in Y$ so that $\tilde{W}(p) \in \pi_{Y}(\mathscr{U})$. Thus $\pi_{Y}^{-1}(\tilde{W}(p)) \in \mathscr{U}$ and

$$
\pi_{Y}^{-1}(\tilde{W}(p)) \subset W(K \times\{p\}) \subset \bigcup\{V(x, p): x \in F(p)\} .
$$

It follows that some member of the finite collection $\{V(x, p): x \in F(p)\}$ belongs to $\mathscr{U}$, and so $\mathscr{U}$ is a Cauchy filter.

In considering the next proposition, it is interesting to note that since (FT-) almost precompactness is a regular-closed hereditary property that is not closed hereditary, the condition of Proposition 3.10(b) that $f$ be open or irreducible cannot be omitted [10, Theorem 3.7.29]. 
Proposition 3.10. Let $X$ and $Y$ be spaces and let $f$ be a continuous map from $X$ onto $Y$.

(a) If $X$ is (FT-) almost precompact, so is $Y$.

(b) If $f$ is a perfect map that is open or irreducible and $Y$ is (FT-) almost precompact, $X$ is (FT-) almost precompact.

Proof. (a) Suppose that $X$ is (FT-) almost precompact and let $W$ be a member of the fine (transitive) quasi-uniformity of $Y$. Since $f$ is continuous, $f:(X, \mathscr{F} \mathscr{T}) \rightarrow(Y, \mathscr{F} \mathscr{T})$ and $f:(X, \mathscr{F} \mathscr{I} \mathscr{N} \mathscr{E}) \rightarrow(Y, \mathscr{F} \mathscr{I} \mathscr{N} \mathscr{E})$ are quasi-uniformly continuous [13, Proposition 2.17]. Thus there exists a $V$ in $\mathscr{F} \mathscr{T}(\mathscr{F} \mathscr{I} \mathscr{N} \mathscr{E})$ so that if $(a, b) \in V$, then $(f(a), f(b)) \in W$. There is a finite subset $F$ of $X$ so that $\overline{V(F)}=X$. It is easily verified that $\overline{W(f(F))}$ $=Y$.

(b) We first suppose that $f$ is an irreducible map and that $Y$ is (FT-) almost precompact. Let $\mathscr{F}$ be an open ultrafilter over $X$. Since $f$ is a closed irreducible map, $\mathscr{H}=\{$ int $f(G): G \in \mathscr{F}\}$ is an open ultrafilter over $Y$. Let $W$ and $V$ be open neighbornets belonging to the fine (transitive) quasiuniformity of $X$ so that $V^{2} \subset W$. Set

$$
\tilde{V}=\left\{(a, b) \in Y \times Y: f^{-1}(b) \subset V\left(f^{-1}(a)\right)\right\} .
$$

Since $f$ is a closed map, $\tilde{V}$ belongs to the fine (transitive) quasi-uniformity of $Y$, and there exists $p \in Y$ so that $\tilde{V}(p) \in \mathscr{H}$. There is a finite subset $A$ of $X$ so that $f^{-1}(p) \subset V(A)$. Thus $f^{-1}(\tilde{V}(p)) \subset V\left(f^{-1}(p)\right) \subset W(A)$. Because $\tilde{V}(p)$ is an open set belonging to $\mathscr{H}, f^{-1}(\tilde{V}(p)) \in \mathscr{F}$ and so, for some $a \in A, W(a) \in \mathscr{F}$.

We now suppose that $f$ is an open map and that $Y$ is (FT-) almost precompact. Let $\mathscr{F}$ be an open ultrafilter over $X$. Then $f(\mathscr{F})$ is an open ultrafilter $\mathscr{H}$ over $Y$. The remainder of the proof follows word-for-word from above.

According to Woods [54], a property $\mathscr{P}$ is a co-absolute invariant property provided that a space $X$ has property $\mathscr{P}$ if and only if the absolute of $X$ has property $\mathscr{P}$.

COROLlaRY (FT-) almost precompactness is a co-absolute invariant property.

The result for pseudocompact spaces corresponding to the previous corollary is proved by Woods [52, Proposition 2.5]. The analogues for pseudocompactness of Propositions 3.9 and 3.10 also obtain. Moreover, 
according to results of Woods [53, Proposition 2.2] and Broverman [3, Theorem 2.3], in order that FINE-pseudocompactness and almost precompactness coincide it is necessary that conditions (b), (c) and (d) of Proposition 3.9 obtain.

Since $T$ is a primary example of an almost precompact space that is not compact, in light of Corollary 3.10, we consider the absolute of this space.

Proposition 3.11. Let $T^{+}=(\mu+1) \cup \omega$ be the one-point compactification of $T$, where $\mu$ is taken to be the point-at-infinity and $\left\{A_{\alpha}: \alpha \in \mu\right\}$ is taken to be the required maximal increasing tower on $\omega$. Define $f: \beta \omega \rightarrow T^{+}$ by $f(n)=n$ for each $n \in \omega$, and if $\mathscr{U} \in \beta \omega-\omega$ define $f(\mathscr{U})=\inf \{\alpha \in \mu$ : $\left.A_{\alpha} \in \mathscr{U}\right\}$ if some $A_{\alpha} \in \mathscr{U}$, and $f(\mathscr{U})=\mu$ if for each $\alpha \in \mu, A_{\alpha} \notin \mathscr{U}$. Then $\beta \omega-f^{-1}(\{\mu\})$ is the absolute of $T$.

Proof. As in 3.12.17 of [10], we see that $f$ is a continuous, and hence perfect, mapping. Moreover, it is easily verified that $f$ is irreducible. It follows that $f \mid \beta \omega-f^{-1}(\{\mu\})$ is a perfect irreducible continuous map of $\beta \omega-f^{-1}(\{\mu\})$ onto $T$. Thus $\beta \omega-f^{-1}(\{\mu\})$ is the absolute of $T$. (See [54, Page 327].)

A free ultrafilter $\mathscr{U}$ over $\omega$ is called a $T$-point provided that $\mathscr{U}$ contains the complement of every member of some maximal increasing tower [39].

COROLlaRY. If $\mathscr{U}$ is a T-point, $\beta \omega-\{\mathscr{U}\}$ is almost precompact.

Proof. Let $\mathscr{U}$ be a $T$-point, and let $\left\{A_{\alpha}: \alpha \in \mu\right\}$ be a maximal increasing tower disjoint from $\mathscr{U}$. We assume that $T$ and $f$ are defined as in the previous proposition. By Corollary 3.10, $\beta \omega-f^{-1}(\{\mu\})$ is almost precompact so that by Proposition 3.9(c) it suffices to note that $\beta \omega-$ $f^{-1}(\{\mu\})$ is a dense subspace of $\beta \omega-\{\mathscr{U}\}$.

Recall that if $X$ is a topological space $X^{*}$ denotes $\beta X-X$. It is known to be consistent that every point of $\omega^{*}$ is a $T$-point (see [2]). $\mathrm{K}$. Kunen has shown that under the continuum hypothesis there exists $p \in \omega^{*}$ such that $p$ is not a $T$-point (and by [39, Corollary 2.8] there are then $2^{\mathfrak{c}}$ such points.) It follows from our next proposition that even if $p$ is not a $T$-point, $\beta \omega-\{p\}$ is almost precompact, (see remarks following Proposition 3.12). The proposition is suggested by results of V. I. Malyhin [35]. 
Proposition 3.12. Let $X$ be a locally compact realcompact space with the Souslin property and let $x \in X^{*}$. Then $\beta X-\{x\}$ is FINE-pseudocompact.

Proof. By Lemma 3.6, it suffices to find an $\omega$-bounded subset $B$ of $X^{*}$ such that $x \in\left(\mathrm{cl}_{X^{*}} B\right)-B$. Since $X$ is realcompact, no point of $X^{*}$ is isolated. Therefore, if $x$ is a $P$-point of $X^{*}$, so that the intersection of any countable family of neighborhoods of $x$ is a neighborhood of $x$, then $X^{*}-\{x\}$ is the required $\omega$-bounded subset. Suppose that $x$ is not a $P$-point of $X^{*}$ and let $H$ be a $G_{\delta}$-set of $X^{*}$ such that $x \in H-$ int $_{X^{*}} H$. There is a countable collection of zero-sets of $X^{*},\left\{Z_{n}: n \in \omega\right\}$, and a countable collection of open sets of $X^{*},\left\{G_{n}: n \in \omega\right\}$, so that $H=$ $\bigcap\left\{G_{n}: n \in \omega\right\}$ and for each $n \in \omega, x \in$ int $_{X^{*}} Z_{n} \subset Z_{n} \subset G_{n}$. Set $Z=$ $\bigcap\left\{Z_{n}: n \in \omega\right\}$ and note that $x \notin$ int $_{X^{*}} Z$. Since $x \in$ int $_{X^{*}} Z_{1}, x \notin$ int $_{Z_{1}} Z$. Define $\Delta=\left\{C \subset Z: C\right.$ is a nonempty cozero-set of $X^{*}$ and $C \subset$ int $\left._{Z_{1}} Z\right\}$ and for each $C \in \Delta$ choose $x_{C} \in C$. Set $H=\left\{x_{C}: C \in \Delta\right\}$. We show that the required $\omega$-bounded set is $H(\omega)=\bigcup\left\{\operatorname{cl}_{X^{*}} C: C\right.$ is a countable subset of $H$ \}. Evidently $H(\omega)$ is $\omega$-bounded.

To see that $x \in \mathrm{cl}_{X^{*}} H(\omega)$, let $G$ be an $X^{*}$-open set about $x$. Recall that $Z$ is a zero-set in the remainder of a locally compact realcompact space, so that by [48, Proposition 4.21], $x \in Z=\mathrm{cl}_{X^{*}}\left(\right.$ int $_{X^{*}} Z$ ). Let $y \in G \cap$ int $_{X^{*}} Z$. There is a cozero-set $R$ so that $y \in R \subset G \cap$ int $_{X^{*}} Z$. Since $R \in \Delta, R \cap H \neq \varnothing$ and so $G \cap H(\omega) \neq \varnothing$.

To see that $x \notin H(\omega)$, let $\left\{x_{C_{n}}: n \in \omega\right\}$ be a countable subset of $H$, where $C_{n} \subset$ int $_{Z_{1}} Z \subset Z_{1}$ and $x_{C_{n}} \in C_{n}$. Then $\mathrm{cl}_{X^{*}}\left(\left\{x_{C_{n}}: n \in \omega\right\}\right) \subset$ $\mathrm{cl}_{X^{*}}\left(\cup\left\{C_{n}: n \in \omega\right\}\right)$ and since $\mathrm{cl}_{X^{*}}\left(\cup\left\{C_{n}: n \in \omega\right\}\right) \subset Z_{1}$,

$$
\mathrm{cl}_{X^{*}}\left(\bigcup\left\{C_{n}: n \in \omega\right\}\right)=\mathrm{cl}_{Z_{1}}\left(\bigcup\left\{C_{n}: n \in \omega\right\}\right) \text {. }
$$

Since $Z_{1}$ is a zero-set in $X^{*}$, by [48, Corollary 1.63], the disjoint cozero-sets $\cup\left\{C_{n}: n \in \omega\right\}$ and $Z_{1}-Z$ have disjoint closures in $Z_{1}$. Thus

$$
\operatorname{cl}_{Z_{1}}\left(\bigcup\left\{C_{n}: n \in \omega\right\}\right) \subset Z_{1}-\operatorname{cl}_{Z_{1}}\left(Z_{1}-Z\right)=\operatorname{int}_{Z_{1}} Z
$$

and so $x \notin \operatorname{cl}_{X^{*}}\left(\left\{x_{C_{n}}: n \in \omega\right\}\right) \subset \operatorname{cl}_{Z_{1}}\left(\cup\left\{C_{n}: n \in \omega\right\}\right)$.

We conclude this section with a few observations concerning $\beta \omega$. First, we note that the space $\beta X$ of the previous proposition is not of countable tightness at $x$. We have just seen that $Y=\beta X-\{x\}$ is FINE-pseudocompact and if $\beta X$ is extremally disconnected at $x$ (see [6, Definition 1.7]), for instance if $x$ is a remote point of $X$, then $Y$ is almost precompact as well. For $Y$ cannot be FINE-complete and so by [13, Corollary 5.32 and Theorem 5.33], for each normal neighbornet $V$ of $Y$ 
there exists $p \in Y$ so that $\operatorname{cl}_{Y} V(p)$ is not compact. Thus $\operatorname{cl}_{Y} V(p) \cup\{x\}$ $=\mathrm{cl}_{\beta X} V(p)$, which is a neighborhood of $x$. It follows that there is a finite set $F$ so that $Y-$ int $_{\beta X} \mathrm{cl}_{\beta X} V(p) \subset V(F)$ and hence

$$
Y=\mathrm{cl}_{Y} V(\{p\} \cup F) \text {. }
$$

Let $p \in \omega^{*}$. There is a marked difference in the behavior of $\beta \omega-\{p\}$ and $\omega^{*}-\{p\}$. Because $\beta \omega-\{p\}$ is separable, it follows from the remarks above that $\beta \omega-\{p\}$ is almost precompact. But if $p$ is a simple $P$-point [38] of the non-separable space $\omega^{*}$, then $p$ has an open-and-closed neighborhood base $\mathscr{G}$ in $\omega^{*}$ that is well ordered by reverse inclusion. Consequently, $\mathscr{C}=\left\{\omega^{*}-G: G \in \mathscr{G}\right\}$ is an interior-preserving open cover of $\omega^{*}-\{p\}$. Hence each $\mathscr{F} \mathscr{T}\left(\omega^{*}-\{p\}\right)$-Cauchy ultrafilter meets $\mathscr{C}$ and so converges. Notice, however, that if $p$ is a point of $\omega^{*}$ and $f$ is an embedding of $\beta \omega$ into $\omega^{*}$, then it follows from Proposition 3.10 and the preceding remarks that $f(\beta \omega)-\{f(p)\}$ is a closed almost precompact subspace of $\omega^{*}-\{f(p)\}$. Thus, $\omega^{*}-\{f(p)\}$ is not FINE-complete.

4. $\Sigma$-products and explicit constructions of extensions. The primary purpose of this section is to show that for $\mathscr{P}=$ FINE-completeness, the maximal $P$-extension of certain $\Sigma$-products is their corresponding Tychonoff product. In pursuing this purpose, we shall also show that any $\Sigma$-product with compact factors is almost precompact.

Proposition 4.1. Let $A$ be a dense weakly Lindelöf subspace of a space $X$. If $A$ is contained in an $\omega$-bounded subspace of $X$, then $X$ is almost precompact.

Proof. Let $\mathscr{G}$ be an open ultrafilter over $X$, let $V$ be an open normal neighbornet and let $W$ be an open neighbornet so that $W^{2} \subset V$. There is a countable subset $D$ of $A$ so that $A \subset \overline{W(D)}$. Since $W(D)$ is an open dense set, $W(D) \in \mathscr{G}$. There is a finite set $E$ so that $D \subset \bar{D} \subset W(E)$. Thus $V(E) \in \mathscr{G}$ and there is an $x \in E$ so that $V(x) \in \mathscr{G}$.

COROLlaRY. Let $B$ be an w-bounded weakly Lindelöf subspace of a FINE-complete space. Then $\bar{B}$ is compact.

Following A. V. Arhangel'skii and D. V. Ranchin [1], we adopt the following notation. If $Y=\Pi\left\{X_{\alpha}: \alpha \in A\right\}$ is the uncountable Cartesian product of nondegenerate topological spaces, the $\sigma$-product of the spaces $X_{\alpha}$ with base point $p \in Y$, which is denoted by $\sigma(Y, p)$, is the subspace of the $\Sigma$-product of the spaces $X_{\alpha}$ with base point $p$, which is defined as 
follows:

$$
\sigma(Y, p)=\{x \in Y: x(\alpha) \neq p(\alpha) \text { for only finitely many } \alpha \in A\} .
$$

It is noted in [1, Remark 2] that if $Y$ is the product of compact spaces then $\sigma(Y, p)$ is a dense $\sigma$-compact subspace of $\Sigma(Y, p)$.

Proposition 4.2. Let $\Sigma(Y, p)$ be a $\Sigma$-product of compact spaces. Then $\Sigma(Y, p)$ is almost precompact. In fact, any Tychonoff product of such spaces is almost precompact.

Proof. It is well known that $\Sigma(Y, p)$ is $\omega$-bounded and since $\sigma(Y, p)$ is a dense Lindelöf subspace, by Proposition $4.1, \Sigma(Y, p)$ is almost precompact. Now let $X=\Pi\left\{\Sigma_{\alpha}: \alpha \in A\right\}$ be a Tychonoff product, where for each $\alpha \in A, \Sigma_{\alpha}$ is a $\Sigma$-product with compact factors. If $A^{\prime}$ is a finite subset of $A$ then $\Pi\left\{\Sigma_{\alpha}: \alpha \in A^{\prime}\right\}$ contains a dense $\sigma$-compact subspace and so is weakly Lindelöf. It follows that $X$ is a weakly Lindelöf $\omega$-bounded space [45, Theorem 1.3]. Once again, Proposition 4.1 obtains. $\square$

Corollary. Let $\alpha$ be an uncountable cardinal and let $\overline{0}: \alpha \rightarrow 2$ be the constant function $\overline{0}(\beta)=0$ and let $\overline{1}: \alpha \rightarrow 2$ be the constant function $\overline{1}(\beta)=1, \beta<\alpha$. Then $2^{\alpha}-\{\overline{0}\}$ is almost precompact.

Proof. By Proposition 4.2, $\Sigma\left(2^{\alpha}, \overline{1}\right)$ is a dense almost precompact subspace of $2^{\alpha}-\{\overline{0}\}$ and so Proposition 3.9(c) obtains.

In [42], Scott proved that no $\Sigma$-product is orthocompact and in [26], G. I. Kac proved that if $Y$ is an uncountable product of spaces with at least two points and $p \in Y$, then $Y-\{p\}$ is not Dieudonné complete. Our next proposition generalizes these results.

Proposition 4.3. Let $X=\Pi\left\{X_{\alpha}: \alpha \in A\right\}$ be an uncountable product of spaces with at least two points and let $p \in X$. If $Z=\Sigma(X, p)$ or $Z=X-\{p\}$, then $Z$ is not FINE-complete.

Proof. Let $\Sigma(2)=\Sigma\left(2^{A}, \overline{0}\right)$. Then $Z$ contains a closed homeomorphic copy of the almost precompact non-compact space $\Sigma(2)$, or $Z$ contains a closed homeomorphic copy of the almost precompact non-compact space $2^{A}-\{\overline{0}\}$.

We have seen that no $\Sigma$-product is FINE-complete and yet it is often the case that the corresponding Tychonoff product enjoys one or more of the completeness properties we have been investigating. For example, the 
product of weakly orthocompact spaces is FT-complete, the product of metacompact spaces is PF-complete, and, of course, all extension properties (in particular all our completeness properties) are productive. We exploit the difference in the behavior of $\Sigma$-properties and Tychonoff products to show that the Tychonoff product often provides the maximal extension of a corresponding $\Sigma$-product.

Proposition 4.4. Let $\Pi=\Pi\left\{X_{\alpha}: \alpha \in A\right\}$ be an uncountable product of spaces with at least two points and let $\Sigma=\Sigma(\Pi, p)$ for some $p \in \Pi$. Then $\Sigma$ is $C^{*}$-embedded in $\Pi$ if and only if, whenever $Y$ is a FINE-complete space and $f: \Sigma \rightarrow Y$ is a continuous function, $f$ admits a continuous extension $\tilde{f}: \Pi \rightarrow Y$.

Proof. Suppose that $\Sigma$ is $C^{*}$-embedded in $\Pi$, let $Y$ be a FINE-complete space and let $f: \Sigma \rightarrow Y$ be a continuous map. Since $\Sigma$ is $C^{*}$-embedded in $\Pi, f$ admits a continuous extension $\tilde{f}: \Pi \rightarrow \beta Y$. We show that, in fact, that $\tilde{f}(\Pi) \subset Y$. For each $\alpha \in A$ let $q_{\alpha} \in X_{\alpha}$ so that $q_{\alpha} \neq p(\alpha)$. Let $x \in \Pi$ and define $x^{\prime} \in \Pi$ as follows: $x^{\prime}(\alpha)=x(\alpha)$ if $x(\alpha) \neq p(\alpha)$ and $x^{\prime}(\alpha)=q_{\alpha}$ if $x(\alpha)=p(\alpha)$. Let $\Sigma(2)=\Sigma\left(\Pi\left(\left\{x^{\prime}(\alpha), p(\alpha)\right\}\right), p\right)$, and let $\hat{f}$ denote $f \mid \Sigma(2)$. Note that $\Sigma(2)$ has the Souslin property, that $\hat{f}(\Sigma(2))=$ $f(\Sigma(2)) \subset f(\Sigma) \subset Y$ and that $x \in \Pi\left\{x^{\prime}(\alpha), p(\alpha)\right\}=\beta \Sigma(2)[18$, Theorem 2]. By Lemma 3.6, $\hat{f}$ admits a continuous extension $h$ mapping $\Sigma(2) \cup\{x\}$ $\rightarrow Y$. As $h$ agrees with $\tilde{f}$ on $\Sigma(2), \tilde{f}(x)=h(x) \in Y$.

Proposition 4.4 generalizes a well-known result of $\mathrm{M}$. Ulmer, namely that a $\Sigma$-product is $C^{*}$-embedded in its corresponding Tychonoff product if and only if it is $C$-embedded in this product [46, Corollary 2.10]. In light of Proposition 4.4, we wish to know under what conditions a $\Sigma$-product is $C$-embedded in its corresponding Tychonoff product. In $\$ 3$ of [46], it is shown that a $\Sigma$-product is not always so embedded even when all the factor spaces are realcompact. Recall that a space $X$ is pseudo- $\boldsymbol{\aleph}_{1}$-compact provided that each locally finite open collection of $X$ is countable. Evidently, a space that is either pseudocompact or weakly Lindelöf is pseudo- $\boldsymbol{\aleph}_{1}$-compact. Some interesting cases in which a $\Sigma$-product is $C$-embedded in its corresponding Tychonoff product are instanced by the following result of $\mathrm{M}$. Ulmer [46, Theorem 2.2]: If a $\Sigma$-product is pseudo- $\boldsymbol{\aleph}_{1}$-compact, or if each factor space of the corresponding Tychonoff product is first countable, or if each factor space of the corresponding Tychonoff product is a $P$-space, then the $\Sigma$-product is $C$-embedded in the corresponding Tychonoff product. 
For notational convenience, if $\mathscr{P}=$ PF-completeness, LF-completeness, SC-completeness, FT-completeness or FINE-completeness, we denote the maximal $P$-extension $\mathscr{P}(X)$ of a space $X$ by $\beta_{\mathrm{PF}}(X), \beta_{\mathrm{LF}}(X)$, and so forth. Recall that, since SC-completeness is almost realcompactness, $\beta_{\mathrm{SC}} X$ is the almost realcompact extension $a X$ introduced by Woods in [55]. In light of results from $\S 2$, the referee suggests that it would be interesting to determine conditions under which either $\beta_{\mathrm{LF}}(X)$ or $\beta_{\mathrm{PF}}(X)$ coincide with $a X$.

Proposition 4.5. Let $\Pi$ be a product of PF-complete, (LF-complete, SC-complete, FT-complete or FINE-complete) spaces and let $X$ be a corresponding $\Sigma$-product that is $C^{*}$-embedded in $\Pi$. Then $\Pi=\beta_{\mathrm{PF}}(X), \beta_{\mathrm{LF}}(X)$, $\beta_{\mathrm{SC}}(X), \beta_{\mathrm{FT}}(X)$ or $\beta_{\mathrm{FINE}}(X)$.

COROLlaRy ([18]). Let $\Sigma$ be a $\Sigma$-product with compact factors and let $\Pi$ be the corresponding Tychonoff product. Then $\Pi=\beta_{\mathrm{FINE}}(\Sigma)=\beta(\Sigma)$.

COROLLARY ([5]). Let $\Sigma$ be a $\Sigma$-product whose factors are separable metrizable spaces and let $\Pi$ be the corresponding Tychonoff product. Then $\Pi=\beta_{\mathrm{FINE}}(\Sigma)=v(\Sigma)$.

We now turn to problems concerning products of FINE-pseudocompact spaces, in which our results concerning $\Sigma$-products play a role.

Proposition 4.6. Let $X$ and $Y$ be FINE-pseudocompact spaces and suppose that $X \times Y$ is pseudocompact. Then $X \times Y$ is FINE-pseudocompact.

Proof. Since $X \times Y$ is pseudocompact, $\beta(X \times Y)=\beta X \times \beta Y$ and so, by a result of $\mathrm{W}$. W. Comfort and $\mathrm{H}$. Herrlich [4, Theorem 2.2], $\beta_{\mathrm{FINE}}(X \times Y)=\beta_{\mathrm{FINE}}(X) \times \beta_{\mathrm{FINE}}(Y)=\beta(X) \times \beta(Y)=\beta(X \times Y)$.

The following example shows that we cannot omit the hypothesis of the preceding proposition that $X \times Y$ is pseudocompact.

EXAMPLE 4.7. There are two almost precompact spaces $X_{1}$ and $X_{2}$ so that $X_{1} \times X_{2}$ is not pseudocompact. Let $X_{1}$ and $X_{2}$ be the spaces of [41, Example 5.2]. These spaces, which are constructed from two types of weak $P$-points, are separable subspaces of $\beta \omega$ with the property that for $i=1,2$, each $X_{i} \cap \omega^{*}$ is an $\omega$-bounded subset of $\omega^{*}$. As shown in [41], $X_{1}$ and $X_{2}$ are countably compact and $X_{1} \times X_{2}$ contains an open-and-closed 
infinite discrete subspace. To see that $X_{1}$ and $X_{2}$ are almost precompact, let $V_{i}$ be an open neighbornet in $X_{l}$ and let $W_{i}$ be an open neighbornet in $X_{i}$ so that $W_{i}^{2} \subset V_{i}$. By Corollary 3.3, there is a finite subset $E_{i}$ of $X_{i}$ so that $W_{l}\left(\omega^{*} \cap X_{i}\right) \subset \operatorname{cl}_{X_{t}}\left(V_{l}\left(E_{l}\right)\right)$. Since each $X_{t}$ is countably compact it follows that each $X_{l}$ is almost precompact.

Our last proposition is related to the following theorem of I. Glicksberg [18, page 370]: If every countable subproduct of a product space is pseudocompact, then the product space is pseudocompact. Using Glicksberg's theorem we establish an analogous result for FINE-pseudocompactness.

Proposition 4.8. Let $X=\Pi\left\{X_{\alpha} \mid \alpha \in A\right\}$ be a product space, where each $X_{\alpha}$ has at least two points, and suppose that for each countable subset I of $A, \Pi\left\{X_{\alpha}: \alpha \in I\right\}$ is FINE-pseudocompact. Then $X$ is FINE-pseudocompact.

Proof. Since

$$
\begin{aligned}
\prod\left\{\beta_{\mathrm{FINE}}\left(X_{\alpha}\right): \alpha \in A\right\} & =\prod\left\{\beta X_{\alpha}: \alpha \in A\right\} \\
& =\beta\left(\prod\left\{X_{\alpha}: \alpha \in A\right\}\right)=\beta X,
\end{aligned}
$$

it suffices to show that $\beta_{\mathrm{FINE}}(X)=\Pi\left\{\beta_{\mathrm{FINE}}\left(X_{\alpha}\right): \alpha \in A\right\}$. Let $Q$ be a FINE-complete space and let $f: \Pi\left\{X_{\alpha}: \alpha \in A\right\} \rightarrow Q$. Evidently $\Pi\left\{X_{\alpha}: \alpha \in A\right\}$ is a dense subspace of the FINE-complete space $\Pi\left\{\beta_{\text {FINE }}\left(X_{\alpha}\right): \alpha \in A\right\}$ and so we need only show that $f$ admits a continuous extension $\tilde{f}: \Pi\left\{\beta_{\mathrm{FINE}}\left(X_{\alpha}\right): \alpha \in A\right\} \rightarrow Q$. Set $D=\{x \in$ $\Pi\left\{\beta_{\text {FINE }}\left(X_{\alpha}\right): \alpha \in A\right\}: x(\alpha) \in X_{\alpha}$ for all but countably many $\left.\alpha \in A\right\}$. Let $f^{\beta}: \beta X \rightarrow \beta Q$ be the continuous extension of $f$ to $\beta X$. Then $D \subset$ $\Pi\left\{\beta_{\text {FINE }}\left(X_{\alpha}\right): \alpha \in A\right\}=\beta X$. We first show that $f^{\beta}(D) \subset Q$. Let $d \in D$. There is a countable subset $I$ of $A$ so that if $\alpha \in A-I$ then $d(\alpha) \in X_{\alpha}$. Let $X^{\prime}=\Pi\left\{X_{\alpha}^{\prime}: \alpha \in A\right\}$ where $X_{\alpha}^{\prime}=X_{\alpha}$ if $\alpha \in I$ and $X_{\alpha}^{\prime}=\{d(\alpha)\}$ if $\alpha \in A-I$. By definition, $f \mid X^{\prime}$ has a continuous extension $g: \beta_{\mathrm{FINE}} X^{\prime} \rightarrow$ $Q$, and since $X^{\prime}$ is FINE-pseudocompact

$$
\beta_{\mathrm{FINE}} X^{\prime}=\beta X^{\prime}=\prod\left\{\beta X_{\alpha}^{\prime}: \alpha \in A\right\} \subset \prod\left\{\beta X_{\alpha}: \alpha \in A\right\} .
$$

Since $g$ and $f^{\beta}$ agree on the dense subspace $X^{\prime}$ of $\beta_{\mathrm{FINE}} X^{\prime}, f^{\beta}(d)=$ $g(d) \in Q$.

Now let $x \in \Pi\left\{\beta_{\mathrm{FINE}}\left(X_{\alpha}\right): \alpha \in A\right\}-D$. Let $y \in \Pi\left\{X_{\alpha}: \alpha \in A\right\}$ so that for each $\alpha \in A, y(\alpha) \neq x(\alpha)$. Let $P=\Pi\{\{x(\alpha), y(\alpha)\}: \alpha \in A\}$ and let $S=\Sigma(P, y)$. Note that $S$ is an $\omega$-bounded subspace of $D$ and that $\beta S=P$. Since $x \in P$ and $S$ has the Souslin property, $f^{\beta} \mid S$ admits a 
continuous extension $h: S \cup\{x\} \rightarrow Q$. Since $h$ agrees with $f^{\beta}$ on $S$, $f^{\beta}(x)=h(x) \in Q$. Thus the range of $f^{\beta}$ is a subset of $Q$, as required.

Question 4.9. Let $\left\{X_{i}: i \in \omega\right\}$ be a countable collection of FINEpseudocompact spaces such that $X=\Pi\left\{X_{i}: i \in \omega\right\}$ is pseudocompact. Is $X$ necessarily FINE-pseudocompact?

Acknowledgment. The first author gratefully acknowledges the support of the Swiss National Science Foundation.

\section{REFERENCES}

[1] A. V. Arhangel'skii and D. V. Ranchin, Everywhere-dense subspaces of topological products and properties related to final compactness, Vestnik Moskov Univ. Mat., (= Moscow Univ. Bull.) 37 (1982), 21-28.

[2] B. Balcar, R. Frankiewicz, and C. Mills, More on nowhere dense closed P-sets, Bull. Acad. Polon. Sci. Ser. Math. Astron. Phys., 28 (1980), 295-299.

[3] S. Broverman, Pseudocompactness properties, Proc. Amer. Math. Soc., 59 (1976), 175-178.

[4] W. W. Comfort and H. Herrlich, On the relations $P(X \times Y)=P(X) \times P(Y)$, General Topology Appl., 6 (1976), 37-43.

[5] H. H. Corson, Normality in subspaces of product spaces, Amer. J. Math., 81 (1959), 785-796.

[6] E. van Douwen, Remote points, Dissertationes Math., 188 (1981) Warsaw.

[7] E. K. van Douwen, The Integers and Topology, Handbook of Set-Theoretic Topology, ed. K. Kunen and J. Vaughan, North Holland, (1984), 111-167.

[8] N. Dykes, Mappings and realcompact spaces, Pacific J. Math., 31 (1969), 347-358.

[9] N. Dykes, Generalizations of realcompact spaces, Pacific J. Math., 33 (1970), 571-581.

[10] R. Engelking, General Topology, Monografie Mat., 60, Polish Scientific Publishers, Warsaw, 1977.

[11] W. M. Fleischman, A new extension of countable compactness, Fund. Math., 67 (1970), 1-9.

[12] _ On fundamental open coverings, Proc. International Conference on Topology and its Applications, Herceg Novi, Yugoslavia, 1968.

[13] P. Fletcher and W. F. Lindgren, Quasi-Uniform Spaces, Lecture Notes in Pure and Applied Mathematics, 77 (1982), Marcel Dekker, New York.

[14] , Quasi-uniformities with a transitive base, Pacific J. Math., 43 (1972), 619-631.

[15] P. Fletcher and S. A. Naimpally, On almost-complete and almost-precompact quasiuniform spaces, Czech. Math. J., 21(96) (1971), 383-390.

[16] S. P. Franklin and M. Rajagopalan, Some examples in topology, Trans. Amer. Math. Soc., 155 (1971), 305-314.

[17] Z. Frolik, A generalization of realcompact spaces, Czech Math. J., 13(88) (1963), 127-138.

[18] I. Glicksberg, Stone- $\breve{C} e c h$ compactifications of products, Trans. Amer. Math. Soc., 90 (1959), 369-382.

[19] S. L. Gulden, W. M. Fleischman and J. H. Weston, Linearly ordered topological spaces, Proc. Amer. Math. Soc., 24 (1970), 197-203.

[20] K. Hardy, Notes on two generalizations of almost realcompact spaces, Math. Centrum Amsterdam Afd. Zuivere Wisk., ZW 57/75, (1975). 
[21] K. P. Hart, Strong collectionwise normality and M. E. Rudin's Dowker space, Proc. Amer. Math. Soc., 83 (1981), 802-806.

[22] R. Haydon, On compactness in spaces of measures and measurecompact spaces, Proc. London Math. Soc., (3) (29) (1974), 1-16.

[23] S. H. Hechler, On some weakly compact spaces and their products, General Topology Appl., 5 (1975), 83-93.

[24] H. Herrlich and J. van der Slot, Properties which are closely related to compactness, Indag. Math., 29 (1967), 524-529.

[25] J. G. Horne, Countable paracompactness and cb-spaces, Notices Amer. Math. Soc., 6 (1959), 629-630.

[26] G. I. Kac, On completely regular spaces withcut complete uniform structures, Uspehi Mat. Nauk (N.S.) (Russian Math. Surveys), 12 (1957), 3(75), 329-332.

[27] A. Kato, Union of realcompact spaces and Lindelöf spaces, Canad. J. Math., 31 (1979), 1247-1268.

[28] J. Keesling, Normality and properties related to compactness in hyperspaces, Proc. Amer. Math. Soc., 24 (1970), 760-766.

[29] P. Kenderov, On Q-spaces, Dokl. Akad. Nauk SSSR, 175 (1967), Soviet Math. Dokl., 8 (1967), 849-852.

[30] H. P. Künzi, A note on Ralph Fox's $\gamma$-space, Proc. Amer. Math. Soc., 91 (1984), 467-470.

[31] H. P. Künzi and P. Fletcher, Even covering properties and somewhat normal spaces, Canad. Math. Bull., to appear.

[32] __ Some generalizations of compactness, submitted for publication.

[33] J. Mack, On a class of countably paracompact spaces, Proc. Amer. Math. Soc., 16 (1965), 467-472.

[34] J. Mack and D. Johnson, The Dedekind completion of $C(X)$, Pacific J. Math., 20 (1967), 231-243.

[35] V. I. Malyhin, On countable spaces having no bicompactification of countable tightness, Dokl. Akad. Nauk SSSR, 206 (1972), 1293-1296 (Soviet Math. Dokl. 13 (1972), 1407-1411.)

[36] M. J. Mansfield, Some generalizations of full normality, Trans. Amer. Math. Soc., 86 (1957), 489-505.

[37] R. L. Moore, Foundations of point-set theory, Amer. Math. Soc. Publ. No. 13, revised edition, 1962.

[38] P. J. Nyiko:, Simple P-points and related matters, preprint.

[39] P. J. Nyikos and J. E. Vaughan, Ordinal extensions of $\omega$ and sequential compactness, preprint.

[40] J. R. Porter and R. G. Woods, Extensions of Hausdorff spaces, Pacific J. Math., 103 (1982), 111-134.

[41] V. Saks, Products of countably compact spaces, Topology Proc., 4 (1979), 553-575.

[42] B. M. Scott, Toward a product theory for orthocompactness, Studies in topology (Proc. Conf. Univ. North Carolina, Charlotte, N. C., 1974; dedicated to Math. Sect. Polish Acad. Sci.), 517-537, Academic Press, New York, 1975.

[43] _ Pseudocompact metacompact spaces are compact, Topology Proceedings, 4 (1979), 577-587.

[44] P. Simon, A note on Rudin's example of Dowker space, Comment. Math. Univ. Carolinae, 12 (1971), No. 4, 825-834.

[45] M. Ulmer, Products of weakly-ぶ-compact spaces, Trans. Amer. Math. Soc., 170 (1972), 279-284.

[46] _ C-embedded $\Sigma$-spaces, Pacific J. Math., 46 (1973), 591-602. 
[47] J. E. Vaughan, Countably Compact and Sequentially Compact Spaces, Handbook of Set-Theoretic Topology, ed. K. Kunen and J. Vaughan, North Holland, (1984), 569-602.

[48] R. C. Walker, The Stone-Čech Compactification, Ergebnisse der Mathematik und ihrer Grenzgebiete, 83 (1974), Springer-Verlag, New York.

[49] M. D. Weir, Hewitt-Nachbin Spaces, Mathematics Studies, 17 (1975) North Holland, Amsterdam.

[50] R. F. Wheeler, Topological measure theory for completely regular spaces and their projective covers, Pacific J. Math., 82 (1979), 565-584.

[51] R. G. Woods, Some $\aleph_{0}$-bounded subsets of Stone-ČCh compactifications, Israel J. Math., 9 (1971), 250-256.

[52] _ Ideals of pseudocompact regular closed sets and absolutes of Hewitt realcompactifications, General Topology Appl., 2 (1972), 315-331.

[53] , Topological extension properties, Trans. Amer. Math. Soc., 210 (1975), 365-385.

[54] _ A survey of absolutes of topological spaces, Topological Structures II, Math. Centre Tracts, 116 (1979), 323-362.

[55] A A Tychonoff almost realcompactification, Proc. Amer. Math. Soc., 43 (1974), 200-208.

[56] P. Zenor, Certain subsets of products of metacompact spaces and subparacompact spaces are realcompact, Canad. J. Math., 24 (1972), 825-829.

Received March 21, 1984 and in revised form May 25, 1984.

UNIVERSITÄT BERN

CH-3012 BERN, SwitzerLAND

AND

VIRGINIA TECH

BLACKSBURG, VA 24061, USA 


\section{PACIFIC JOURNAL OF MATHEMATICS EDITORS}

\author{
V. S. VARADARAJAN (Managing Editor) \\ University of California \\ Los Angeles, CA 90024 \\ Charles R. DePrima \\ California Institute of Technology \\ Pasadena, CA 91125 \\ R. FINN \\ Stanford University \\ Stanford, CA 94305
}

\author{
HeRmanN FlaschKa \\ University of Arizona \\ Tucson, AZ 857.21
}

RAMESH A. GANGOLli

University of Washington

Seattle, WA 98195

ROBION KIRBY

University of California

Berkeley, CA 94720
C. C. MOORE

University of California

Berkeley, CA 94720

H. SAMELSON

Stanford University

Stanford, CA 94305

HAROLD STARK

University of California, San Diego

La Jolla, CA 92093

\section{ASSOCIATE EDITORS}
R. ARENS
E. F. BECKENBACH
B. H. NeumanN
F. WOLF
K. YoSHIDA
(1906-1982)

\section{SUPPORTING INSTITUTIONS}

\begin{abstract}
UNIVERSITY OF ARIZONA
UNIVERSITY OF BRITISH COLUMBIA

CALIFORNIA INSTITUTE OF TECHNOLOGY

UNIVERSITY OF CALIFORNIA

MONTANA STATE UNIVERSITY

UNIVERSITY OF NEVADA, RENO

NEW MEXICO STATE UNIVERSITY

OREGON STATE UNIVERSITY
\end{abstract}

\author{
UNIVERSITY OF OREGON \\ UNIVERSITY OF SOUTHERN CALIFORNIA \\ STANFORD UNIVERSITY \\ UNIVERSITY OF HAWAII \\ UNIVERSITY OF TOKYO \\ UNIVERSITY OF UTAH \\ WASHINGTON STATE UNIVERSITY \\ UNIVERSITY OF WASHINGTON
}

The Supporting Institutions listed above contribute to the cost of publication of this Journal, but they are not owners or publishers and have no responsibility for its content or policies.

Mathematical papers intended for publication in the Pacific Journal of Mathematics should be in typed form or offset-reproduced (not dittoed), double spaced with large margins. Please do not use built up fractions in the text of the manuscript. However, you may use them in the displayed equations. Underline Greek letters in red, German in green, and script in blue. The first paragraph must be capable of being used separately as a synopsis of the entire paper. In particular it should contain no bibliographic references. Please propose a heading for the odd numbered pages of less than 35 characters. Manuscripts, in triplicate, may be sent to any one of the editors. Please classify according to the scheme of Math. Reviews, Index to Vol. 39. Supply name and address of author to whom proofs should be sent. All other communications should be addressed to the managing editor, or Elaine Barth, University of California, Los Angeles, California 90024.

There are page-charges associated with articles appearing in the Pacific Journal of Mathematics. These charges are expected to be paid by the author's University, Government Agency or Company. If the author or authors do not have access to such Institutional support these charges are waived. Single authors will receive 50 free reprints; joint authors will receive a total of 100 free reprints. Additional copies may be obtained at cost in multiples of 50 .

The Pacific Journal of Mathematics is issued monthly as of January 1966. Regular subscription rate: $\$ 190.00$ a year (5 Vols., 10 issues). Special rate: $\$ 66.00$ a year to individual members of supporting institutions.

Subscriptions, orders for numbers issued in the last three calendar years, and changes of address should be sent to Pacific Journal of Mathematics, P.O. Box 969, Carmel Valley, CA 93924, U.S.A. Old back numbers obtainable from Kraus Periodicals Co., Route 100, Millwood, NY 10546.

The Pacific Journal of Mathematics at P.O. Box 969, Carmel Valley, CA 93924 (ISSN 0030-8730) publishes 5 volumes per year. Application to mail at Second-class postage rates is pending at Carmel Valley, California, and additional mailing offices. Postmaster: Send address changes to Pacific Journal of Mathematics, P.O. Box 969, Carmel Valley, CA 93924.

PUBLISHED BY PACIFIC JOURNAL OF MATHEMATICS, A NON-PROFIT CORPORATION

Copyright $\odot 1985$ by Pacific Journal of Mathematics 


\section{Pacific Journal of Mathematics}

Vol. 120, No. $2 \quad$ October, 1985

Philip Marshall Anselone and Mike Treuden, Regular operator

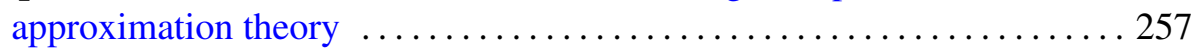

Giuseppe Baccella, Semiprime $\aleph-Q F 3$ rings $\ldots \ldots \ldots \ldots \ldots \ldots \ldots \ldots . \ldots \ldots$

Earl Robert Berkson and Thomas Alastair Gillespie, The generalized M.

Riesz theorem and transference $\ldots \ldots \ldots \ldots \ldots \ldots \ldots \ldots . \ldots 279$

Joachim Boidol, A Galois-correspondence for general locally compact

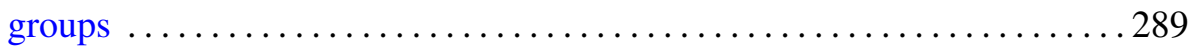

Joseph Eugene D'Atri, Josef Dorfmeister and Yan Da Zhao, The isotropy

representation for homogeneous Siegel domains ............... 295

C. Debiève, On Banach spaces having a Radon-Nikodým dual

Michael Aaron Freedman, Existence of strong solutions to singular

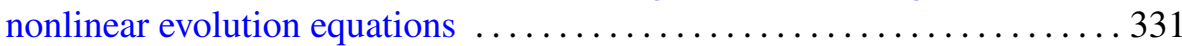

Francisco Jose Freniche, Grothendieck locally convex spaces of continuous

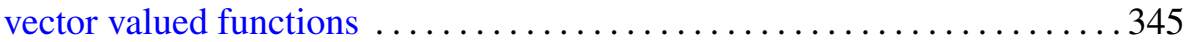

Hans-Peter Künzi and Peter Fletcher, Extension properties induced by complete quasi-uniformities . ............................ 357

Takaŝi Kusano, Charles Andrew Swanson and Hiroyuki Usami, Pairs of

positive solutions of quasilinear elliptic equations in exterior domains . . 385

Angel Rafael Larotonda and Ignacio Zalduendo, Spectral sets as Banach

manifolds

J. Martínez-Maurica and C. Pérez García, A new approach to the

Kreı̆n-Milman theorem

Christian Pommerenke, On the boundary continuity of conformal maps . . . 423

M. V. Subba Rao, Some Rogers-Ramanujan type partition theorems

Stephen Edwin Wilson, Bicontactual regular maps .........

Jaap C. S. P. van der Woude, Characterizations of (H)PI extensions

Kichoon Yang, Deformation of submanifolds of real projective space

Subhashis Nag, Errata: "On the holomorphy of maps from a complex to a

real manifold" 